\title{
A Note on Natural Transformation of Exton's Triple Series Hypergeometric Function
}

\author{
Harsh vardhan Harsh ${ }^{1}$, Puneet Krishna Sharma ${ }^{2}$ and Shabana Khan ${ }^{3}$ \\ ${ }^{1}$ Faculty of Sci. \& Tech., ICFAI Tech. School, ICFAI University Jaipur, Agra Road, Jamdoli, \\ , Jaipur, Rajasthan, India \\ ${ }^{2}$ Department of Applied Mathematics, Amity School of Applied Sciences, Amity University \\ Jaipur, Rajasthan , India \\ ${ }^{3}$ Department of Applied Sciences and Humanities, Faculty of Engineering and Technology, Jamia \\ Millia Islamia University Okhla, New Delhi, India

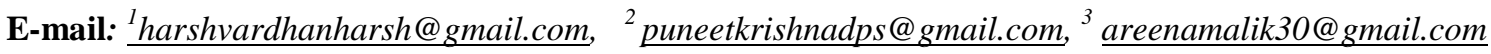

Corresponding Author: Puneet Krishna Sharma

\begin{abstract}
Triple series hypergeometric functions are very important from the applications point of view. Exton had defined 20 triple hypergeometric functions namely $X_{1}, X_{2}, \ldots, X_{20}$. Integral transform technique is widely using for research purpose. Natural transform is a new kind of integral transform and generalization of Laplace transform. In the present research note, we give the Natural integrals of the triple series hypergeometric function due to Exton.
\end{abstract}

Keywords: Hypergeometric functions, Exoton's triple series, $N$-transform.

\section{Introduction}

Triple series hypergeometric functions are well known in literature and large number of applications are available. Srivastava and Kashyap [11] present several interesting applications of hypergeometric series in one and more variable in queuing theory and stochastic process. Lauricella [8] introduced 14 complete set of hypergeometric series in three variables. Saran [9] initiated systematic study of Lauricella set. Srivastava and Karlsson [10] give detailed account on triple series in his famous monograph. In 1982 Exton [2] had defined 20 triple functions namely $X_{1}, X_{2}, \ldots, X_{20}$ in his famous note. In the same note, Laplace integrals of Exoton's series [7] and wide applications are also given which shows importance and applicability of the functions. Integral Transform technique is an important and frequent using tool in mathematical and computational research. Recently developed Natural transform is widely using for application purpose. The natural transformation [3], initially defined by khan and khan as N-transform, who studied properties and applications. Belgacem et. al. [6],[1] defined its inverse and studied some additional fundamental properties of this transform and named it the Natural transform.

\section{Results Required}

The Natural transform [5],[6] $R(s, u)$ of the function for all $t \geq 0$, is given by

$$
\begin{aligned}
& N^{+}[f(t)]=R(s, u)=\frac{1}{u} \int_{0}^{\infty} e^{-s t} f(u t) d t \quad s>0, u>0 \\
& R(s, u)=\frac{1}{u} \int_{0}^{\infty} e^{-\frac{u t}{u}} f(t) d t
\end{aligned}
$$


Harsh Vardhan Harsh, Puneet K. Sharma and Shabana Khan/ A Note on Natural Transformation of Exton's ...

where $t, u$ are time variables and $s$ is the frequency variable, provided the function $f(t)$ is defined in a set

$$
A=\left\{f(t): \exists \tau_{1}, \tau_{2}>0,|f(t)|<M e^{\frac{t}{\tau_{j}}} ; t \in(-1)^{j} \times[0, \infty)\right\} \text {, }
$$

where $M$ is finite constant , $\boldsymbol{\tau}_{\mathbf{1}}, \boldsymbol{\tau}_{\mathbf{2}}$ may be finite or infinite. The discrete form of natural transform [4] is given by

$$
N^{+}[f(t)]=R(s, u)=\sum_{0}^{\infty} \frac{n ! a_{n} u^{n}}{s^{n+1}}
$$

The inverse Natural transformation is defined by

$$
N^{-1}[R(s, u)]=f(t)=\frac{1}{2 \pi i} \int_{c-i \infty}^{c+i \infty} e^{\frac{s t}{u}} R(s, u) d s
$$

By using these definitions, shifted factorial is defined as follows:

$$
(a)_{m}=\frac{\Gamma(a+m)}{\Gamma(a)}=\frac{s}{u} \int_{0}^{\infty} e^{-\frac{s t}{u}}\left(\frac{s t}{u}\right)^{a+m-1} d t
$$

Exton has defined following 20 triple series hypergeometric functions:

$$
\begin{aligned}
& X_{1}(a, b ; c, d ; x, y, z)=\sum_{m, n, p=0}^{\infty} \frac{(a)_{2 m+2 n+p}(b)_{p} x^{m} y^{n} z^{p}}{(c)_{m}(d)_{n+p} m ! n ! p !} \\
& X_{2}\left(a, b ; c_{1}, c_{2}, c_{3} ; x, y, z\right)=\sum_{m, n, p=0}^{\infty} \frac{(a)_{2 m+2 n+p}(b)_{p} x^{m} y^{n} z^{p}}{\left(c_{1}\right)_{m}\left(c_{2}\right)_{n}\left(c_{3}\right)_{p} m ! n ! p !} \\
& X_{3}(a, b ; c, d ; x, y, z)=\sum_{m, n, p=0}^{\infty} \frac{(a)_{2 m+n+p}(b)_{n+p} x^{m} y^{n} z^{p}}{(c)_{m+n}(d)_{p} m ! n ! p !} \\
& X_{4}\left(a, b ; c_{1}, c_{2}, c_{3} ; x, y, z\right)=\sum_{m, n, p=0}^{\infty} \frac{(a)_{2 m+n+p}(b)_{n+p} x^{m} y^{n} z^{p}}{\left(c_{1}\right)_{m}\left(c_{2}\right)_{n}\left(c_{3}\right)_{p} m ! n ! p !} \\
& X_{5}\left(a, b_{1}, b_{2} ; c ; x, y, z\right)=\sum_{m, n, p=0}^{\infty} \frac{(a)_{2 m+n+p}\left(b_{1}\right)_{n}\left(b_{2}\right)_{p} x^{m} y^{n} z^{p}}{(c)_{m+n+p} m ! n ! p !} \\
& X_{6}\left(a, b_{1}, b_{2} ; c, d ; x, y, z\right)=\sum_{m, n, p=0}^{\infty} \frac{(a)_{2 m+n+p}\left(b_{1}\right)_{n}\left(b_{2}\right)_{p} x^{m} y^{n} z^{p}}{(c)_{m+n}(d)_{p} m ! n ! p !} \\
& X_{7}\left(a, b_{1}, b_{2} ; c, d ; x, y, z\right)=\sum_{m, n, p=0}^{\infty} \frac{(a)_{2 m+n+p}\left(b_{1}\right)_{n}(b)_{p} x^{m} y^{n} z^{p}}{(c)_{m}(d)_{n+p} m ! n ! p !}
\end{aligned}
$$


$X_{8}\left(a, b_{1}, b_{2} ; c_{1}, c_{2}, c_{3} ; x, y, z\right)=\sum_{m, n, p=0}^{\infty} \frac{(a)_{2 m+n+p}\left(b_{1}\right)_{n}\left(b_{2}\right)_{p} x^{m} y^{n} z^{p}}{\left(c_{1}\right)_{m}\left(c_{2}\right)_{n}\left(c_{3}\right)_{p} m ! n ! p !}$

$X_{9}(a, b ; c ; x, y, z)=\sum_{m, n, p=0}^{\infty} \frac{(a)_{2 m+n}(b)_{n+2 p} x^{m} y^{n} z^{p}}{(c)_{m+n+p} m ! n ! p !}$

$X_{10}(a, b ; c, d ; x, y, z)=\sum_{m, n, p=0}^{\infty} \frac{(a)_{2 m+n}(b)_{n+2 p} x^{m} y^{n} z^{p}}{(c)_{m+n}(d)_{p} m ! n ! p !}$

$X_{11}(a, b ; c, d ; x, y, z)=\sum_{m, n, p=0}^{\infty} \frac{(a)_{2 m+n}(b)_{n+2 p} x^{m} y^{n} z^{p}}{(c)_{m+p}(d)_{n} m ! n ! p !}$

$X_{12}\left(a, b ; c_{1}, c_{2}, c_{3} ; x, y, z\right)=\sum_{m, n, p=0}^{\infty} \frac{(a)_{2 m+n}(b)_{n+2 p} x^{m} y^{n} z^{p}}{\left(c_{1}\right)_{m}\left(c_{2}\right)_{n}\left(c_{3}\right)_{p} m ! n ! p !}$

$X_{13}(a, b, c ; d ; x, y, z)=\sum_{m, n, p=0}^{\infty} \frac{(a)_{2 m+n}(b)_{n+p}(c)_{p} x^{m} y^{n} z^{p}}{(d)_{m+n+p} m ! n ! p !}$

$X_{14}\left(a, b, c ; d, d^{\prime} ; x, y, z\right)=\sum_{m, n, p=0}^{\infty} \frac{(a)_{2 m+n}(b)_{n+p}(c)_{p} x^{m} y^{n} z^{p}}{(d)_{m+n}\left(d^{\prime}\right)_{p} m ! n ! p !}$

$X_{15}\left(a, b, c ; d, d^{\prime} ; x, y, z\right)=\sum_{m, n, p=0}^{\infty} \frac{(a)_{2 m+n}(b)_{n+p}(c)_{p} x^{m} y^{n} z^{p}}{(d)_{m}\left(d^{\prime}\right)_{n+p} m ! n ! p !}$

$X_{16}\left(a, b, c ; d, d^{\prime} ; x, y, z\right)=\sum_{m, n, p=0}^{\infty} \frac{(a)_{2 m+n}(b)_{n+p}(c)_{p} x^{m} y^{n} z^{p}}{(d)_{m+p}\left(d^{\prime}\right)_{n} m ! n ! p !}$

$X_{17}\left(a, b, c ; d_{1}, d_{2}, d_{3} ; x, y, z\right)=\sum_{m, n, p=0}^{\infty} \frac{(a)_{2 m+n}(b)_{n+p}(c)_{p} x^{m} y^{n} z^{p}}{\left(d_{1}\right)_{m}\left(d_{2}\right)_{n}\left(d_{3}\right)_{p} m ! n ! p !}$

$X_{18}\left(a, b, b^{\prime}, c ; d ; x, y, z\right)=\sum_{m, n, p=0}^{\infty} \frac{(a)_{2 m+n}(b)_{n}\left(b^{\prime}\right)_{p}(c)_{p} x^{m} y^{n} z^{p}}{(d)_{m+n+p} m ! n ! p !}$

$X_{19}\left(a, b, b^{\prime}, c ; d, d^{\prime} ; x, y, z\right)=\sum_{m, n, p=0}^{\infty} \frac{(a)_{2 m+n}(b)_{n}\left(b^{\prime}\right)_{p}(c)_{p} x^{m} y^{n} z^{p}}{(d)_{m}\left(d^{\prime}\right)_{n+p} m ! n ! p !}$

$X_{20}\left(a, b, b^{\prime}, c ; d, d^{\prime} ; x, y, z\right)=\sum_{m, n, p=0}^{\infty} \frac{(a)_{2 m+n}(b)_{n}\left(b^{\prime}\right)_{p}(c)_{p} x^{m} y^{n} z^{p}}{(d)_{m+p}\left(d^{\prime}\right)_{n} m ! n ! p !}$ 


\section{Main Results}

The following twenty interesting integrals involving generalized hypergeometric function will be evaluated in this paper:

1. $X_{1}(a, b ; c, d ; x, y, z)=$

$$
\frac{1}{\Gamma(a)} \frac{1}{\Gamma(b)} \frac{1}{u v} \int_{0}^{\infty} \int_{0}^{\infty} e^{\left(-\frac{s-t}{u}-\frac{t}{v}\right)}\left(\frac{s}{u}\right)^{a-1}\left(\frac{t}{v}\right)^{b-1}{ }_{0} F_{1}\left(-; c ; \frac{x s^{2}}{u^{2}}\right){ }_{0} F_{1}\left(-; d ; y s^{2} u v+z s t u^{2}\right) d s d t
$$

2. $\quad X_{2}\left(a, b ; c_{1}, c_{2}, c_{3} ; x, y, z\right)=\frac{1}{\Gamma(a)} \frac{1}{u} \int_{0}^{\infty} e^{\left(-\frac{s}{u}\right)}\left(\frac{s}{u}\right)^{a-1}{ }_{0} F_{1}\left(-; c_{1} ; \frac{x s^{2}}{u^{2}}\right){ }_{0} F_{1}\left(-; c_{2} ; \frac{y s^{2}}{u^{2}}\right){ }_{1} F_{1}\left(b ; c_{3} ; \frac{z s}{u}\right) d s$

3. $X_{3}(a, b ; c, d ; x, y, z)=$

$$
\frac{1}{\Gamma(a)} \frac{1}{\Gamma(b)} \frac{1}{u v} \int_{0}^{\infty} \int_{0}^{\infty} e^{\left(-\frac{s}{u}-\frac{t}{v}\right)}\left(\frac{s}{u}\right)^{a-1}{ }_{0} F_{1}\left(-; c ; \frac{x s^{2} u v+y t u^{2} s}{u^{2}}\right)\left(\frac{t}{v}\right)^{b-1}{ }_{0} F_{1}\left(-; d ; \frac{z s t}{u v}\right) d s d t
$$

4. $\quad X_{4}\left(a, b ; c_{1}, c_{2}, c_{3} ; x, y, z\right)=\frac{1}{\Gamma(a)} \int_{0}^{\infty} e^{\left(-\frac{s}{u}\right)}\left(\frac{s}{u}\right)^{a-1}{ }_{0} F_{1}\left(-; c_{1} ; x \frac{s^{2}}{u^{2}}\right) \psi_{2}\left(b ; c_{2}, c_{3} ; y \frac{s}{u}, z \frac{s}{u}\right)$

5. $X_{5}\left(a, b_{1}, b_{2} ; c ; x, y, z\right)=$

$$
\frac{1}{\Gamma(a) \Gamma\left(b_{1}\right) \Gamma\left(b_{2}\right)} \frac{1}{u v w} \int_{0}^{\infty} \int_{0}^{\infty} \int_{0}^{\infty} e^{\left(-\frac{s}{u}-\frac{t_{1}}{v}-\frac{t_{2}}{w}\right)}\left(-\frac{s}{u}\right)^{a-1}\left(\frac{t_{1}}{v}\right)^{b_{1}-1}\left(\frac{t_{2}}{w}\right)^{b_{2}-1}{ }_{0} F_{1}\left(-; c ; x\left(\frac{s}{u}\right)^{2}+y\left(\frac{s t_{1}}{u v}\right)+z\left(\frac{s t_{2}}{u w}\right)\right) d s d t_{1} d t_{2}
$$

6. $\quad X_{6}\left(a, b_{1}, b_{2} ; c, d ; x, y, z\right)=\frac{1}{\Gamma(a) \Gamma\left(b_{1}\right)} \frac{1}{u v}$

$$
\times \int_{0}^{\infty} \int_{0}^{\infty} e^{\left(\frac{s}{u}-\frac{t}{v}\right)}\left(\frac{s}{u}\right)^{a-1}\left(\frac{t}{v}\right)^{b_{1}-1}{ }_{0} F_{1}\left(-; c ; \frac{x s^{2}}{u^{2}}+\frac{y s t}{u v}\right){ }_{1} F_{1}\left(b_{2} ; d ; z \frac{s}{u}\right) d s d t
$$

7. $\quad X_{7}\left(a, b_{1}, b_{2} ; c, d ; x, y, z\right)=\frac{1}{u \Gamma(a)} \int_{0}^{\infty} e^{\left(-\frac{s}{u}\right)}\left(\frac{s}{u}\right)^{a-1} \phi_{2}\left(b_{1}, b_{2} ; d ; y \frac{s}{u}, z \frac{s}{u}\right){ }_{0} F_{1}\left(-; c ; x \frac{s^{2}}{u^{2}}\right) d s$

8. $X_{8}\left(a, b_{1}, b_{2} ; c_{1}, c_{2}, c_{3} ; x, y, z\right)=\int_{0}^{\infty} e^{\left(-\frac{s}{u}\right)}\left(\frac{s}{u}\right)^{a-1}{ }_{0} F_{1}\left(-; c_{1} ; x\left(\frac{s}{u}\right)^{2}\right){ }_{1} F_{1}\left(b_{1} ; c_{2} ; y \frac{s}{u}\right){ }_{1} F_{1}\left(b_{2} ; c_{3} ; z \frac{s}{u}\right) d s$

9. $\quad X_{9}(a, b ; c ; x, y, z)=\frac{1}{\Gamma(a) \Gamma(b)} \frac{1}{u v} \int_{0}^{\infty} \int_{0}^{\infty} e^{\left(\frac{s-t}{u}\right)}\left(\frac{s}{u}\right)^{a-1}\left(\frac{t}{v}\right)^{b-1} \sum_{m=0}^{\infty} \frac{1}{(c)_{m}} \frac{\left(x \frac{s^{2}}{u^{2}}+y \frac{s t}{u^{2}}+z \frac{t^{2}}{u v}\right)^{m}}{m !} d s d t$ 
10. $X_{10}(a, b ; c, d ; x, y, z)=$

$$
\frac{1}{u v} \frac{1}{\Gamma(a) \Gamma(b)} \int_{0}^{\infty} \int_{0}^{\infty} e^{\left(-\frac{s}{u}\right)}\left(\frac{s}{u}\right)^{a-1} e^{\left(-\frac{t}{v}\right)}\left(\frac{t}{v}\right)^{b-1}{ }_{0} F_{1}\left(-; c ; x\left(\frac{s}{u}\right)^{2}+y s t\right){ }_{0} F_{1}\left(-; d ; z\left(\frac{t}{v}\right)^{2}\right) d s d t
$$

11. $X_{11}(a, b ; c, d ; x, y, z)=\frac{1}{\Gamma(a) \Gamma(b)} \int_{0}^{\infty} \int_{0}^{\infty} e^{\left(-\frac{s}{u}-\frac{t}{v}\right)}\left(\frac{s}{u}\right)^{a-1}\left(\frac{t}{v}\right)^{b-1}{ }_{0} F_{1}\left(-; c ; x s^{2}+z t^{2}\right)_{0} F_{1}(-; d ; z s t) d s d t$

12. $X_{12}\left(a, b ; c_{1}, c_{2}, c_{3} ; x, y, z\right)=$

$$
\frac{1}{u v} \frac{1}{\Gamma(a) \Gamma(b)} \int_{0}^{\infty} \int_{0}^{\infty} e^{\left(-\frac{s}{u}-\frac{t}{v}\right)}\left(\frac{s}{u}\right)^{a-1}\left(\frac{t}{v}\right)^{b-1}{ }_{0} F_{1}\left(-; c_{1} ; x \frac{s^{2}}{u^{2}}\right){ }_{0} F_{1}\left(-; c_{2} ; y \frac{s t}{u v}\right){ }_{0} F_{1}\left(-; c_{3} ; z \frac{t^{2}}{v^{2}}\right) d s d t
$$

13. $X_{13}(a, b, c ; d ; x, y, z)=$

$$
\frac{1}{\Gamma(a) \Gamma(b) \Gamma(c)} \frac{1}{u v w} \int_{0}^{\infty} \int_{0}^{\infty} \int_{0}^{\infty} e^{\left(-\frac{s_{1}}{u}-\frac{s_{2}}{v}-\frac{s_{3}}{w}\right)}\left(-\frac{s_{1}}{u}\right)^{a-1}\left(\frac{s_{2}}{v}\right)^{b-1}\left(\frac{s_{3}}{w}\right)^{c-1}{ }_{0} F_{1}\left(-; d ; \frac{x s_{1}^{2} w v+y u s_{1} s_{2} w+z u^{2} s_{2} s_{3}}{u^{2} w v}\right) d s_{1} d s_{2} d s_{3}
$$

14. $X_{14}\left(a, b, c ; d, d^{\prime} ; x, y, z\right)=$

$$
\frac{1}{\Gamma(a) \Gamma(b)} \frac{1}{u v} \int_{0}^{\infty} \int_{0}^{\infty} e^{\left(-\frac{s}{u}-\frac{t}{v}\right)}\left(\frac{s}{u}\right)^{a-1}\left(\frac{t}{v}\right)^{b-1}{ }_{0} F_{1}\left(-; d ; \frac{x s^{2} v+y s u t}{u v}\right){ }_{1} F_{1}\left(c ; d^{\prime} ; \frac{z t}{v}\right) d s d t
$$

15. $X_{15}\left(a, b, c ; d, d^{\prime} ; x, y, z\right)=$

$$
\frac{1}{u v} \frac{1}{\Gamma(a) \Gamma(c)} \int_{0}^{\infty} \int_{0}^{\infty} e^{\left(-\frac{s}{u}-\frac{t}{v}\right)}\left(\frac{s}{u}\right)^{a-1}\left(\frac{t}{v}\right)^{c-1}{ }_{0} F_{1}\left(-; d ; x\left(\frac{s}{u}\right)^{2}\right){ }_{1} F_{1}\left(b ; d^{\prime} ; \frac{y s v+z u t}{u v}\right) d s d t
$$

16. $X_{16}\left(a, b, c ; d, d^{\prime} ; x, y, z\right)=$

$$
\frac{1}{\Gamma(a) \Gamma(b) \Gamma(c)} \frac{1}{u v w} \int_{0}^{\infty} \int_{0}^{\infty} \int_{0}^{\infty} e^{\left(-\frac{s_{1}}{u}-\frac{s_{2}}{v}-\frac{s_{3}}{w}\right.}\left(\frac{s_{1}}{u}\right)^{a-1}\left(\frac{s_{2}}{v}\right)^{b-1}\left(\frac{s_{3}}{w}\right)^{c-1}{ }_{0} F_{1}\left(-; d ; \frac{x s_{1}{ }^{2} v w+z u^{2} s_{2} s_{3}}{u^{2} v w}\right){ }_{0} F_{1}\left(-; d^{\prime} ; y \frac{s_{1} s_{2}}{u v}\right) d s_{1} d s_{2} d s_{3}
$$

17. $X_{17}\left(a, b, c ; d_{1}, d_{2}, d_{3} ; x, y, z\right)=$

$$
\frac{1}{\Gamma(a) \Gamma(c)} \int_{0}^{\infty} \int_{0}^{\infty} e^{\left(-\frac{s}{u}-\frac{t}{v}\right)}\left(\frac{s}{u}\right)^{a-1}\left(\frac{t}{v}\right)^{c-1}{ }_{0} F_{1}\left(-; d_{1} ; x \frac{s^{2}}{u^{2}}\right) \Psi_{2}\left(b ; d_{2}, d_{3} ; y \frac{s}{u}, z \frac{t}{v}\right) d s d t
$$

18. $X_{18}\left(a, b, b^{\prime}, c ; d ; x, y, z\right)=$

$$
\frac{1}{u v w t} \frac{1}{\Gamma(a) \Gamma(b) \Gamma\left(b^{\prime}\right) \Gamma(c)} \int_{0}^{\infty} \int_{0}^{\infty} \int_{0}^{\infty} \int_{0}^{\infty} e^{\left(-\frac{s_{1}}{u}-\frac{s_{2}}{v}-\frac{s_{3}}{w}-\frac{s_{4}}{t}\right)}\left(\frac{s_{1}}{u}\right)^{a-1}\left(\frac{s_{2}}{v}\right)^{b-1}\left(\frac{s_{3}}{w}\right)^{b-1}\left(\frac{s_{4}}{t}\right)^{c-1}{ }_{0} F_{1}\left(-; d ; \frac{x s_{1}^{2} w v t+y s_{1} s_{2} u w t+z u^{2} v s_{3} s_{4}}{u^{2} w v t}\right) d s_{1} d s_{2} d s_{3} d s_{4}
$$


19. $X_{19}\left(a, b, b^{\prime}, c ; d, d^{\prime} ; x, y, z\right)=$

$$
\frac{1}{\Gamma(a) \Gamma(c)} \frac{1}{u v} \int_{0}^{\infty} \int_{0}^{\infty} e^{\left(-\frac{s}{u}-\frac{t}{v}\right)}\left(\frac{s}{u}\right)^{a-1}\left(\frac{t}{v}\right)^{c-1}{ }_{0} F_{1}\left(-; d_{1} ; x \frac{s^{2}}{u^{2}}\right) \phi_{2}\left(b, b^{\prime} ; d^{\prime} ; \frac{y s}{u}, \frac{z t}{v}\right) d s d t
$$

20. $X_{20}\left(a, b, b^{\prime}, c ; d, d^{\prime} ; x, y, z\right)=$

$$
\frac{1}{\Gamma(a) \Gamma\left(b^{\prime}\right) \Gamma(c)} \int_{0}^{\infty} \int_{0}^{\infty} \int_{0}^{\infty} e^{\left(-\frac{s_{1}}{u}-\frac{s_{2}}{v}-\frac{s_{3}}{w}\right)}\left(\frac{s_{1}}{u}\right)^{a-1}\left(\frac{s_{2}}{v}\right)^{b^{\prime}-1}\left(\frac{s_{3}}{w}\right)^{c-1}{ }_{0} F_{1}\left(-; d ; \frac{x s_{1}{ }^{2} v w+z u^{2} s_{2} s_{3}}{u^{2} v w}\right){ }_{1} F_{1}\left(b ; d^{\prime} ; \frac{y s_{1}}{u}\right) d s_{1} d s_{2} d s_{3}
$$

\section{Derivation}

In order to derive the main results, first we shall derive the result (3.1), i.e.

$$
\begin{aligned}
& X_{1}(a, b ; c, d ; x, y, z) \\
& =\frac{1}{\Gamma(a) \Gamma(b)} \frac{1}{u v} \int_{0}^{\infty} \int_{0}^{\infty} e^{\left(-\frac{s}{u}-\frac{t}{v}\right)}\left(\frac{s}{u}\right)^{a-1}\left(\frac{t}{v}\right)^{b-1}{ }_{0} F_{1}\left(-; c ; \frac{x s^{2}}{u^{2}}\right){ }_{0} F_{1}\left(-; d ; \frac{y s^{2}}{u^{2}}+\frac{z s t}{u v}\right) d s d t
\end{aligned}
$$

\section{Proof:}

To derive (4.1), we consider L.H.S as I

$$
\begin{aligned}
\mathrm{I} & =X_{1}(a, b ; c, d ; x, y, z)=\sum_{m, n, p=0}^{\infty} \frac{(a)_{2 m+2 n+p}(b)_{p} x^{m} y^{n} z^{p}}{(c)_{m}(d)_{n+p} m ! n ! p !} \\
& =\frac{1}{\Gamma(a) \Gamma(b)} \sum_{m, n, p=0}^{\infty} \frac{x^{m} y^{n} z^{p} p}{(c)_{m}(d)_{n+p} m ! n ! p !} \int_{0}^{\infty} e^{\left(-\frac{s}{u}\right)}\left(\frac{s}{-}\right)^{2 m+2 n+p+a-1}\left(\frac{d s}{u}\right) \int_{0}^{\infty} e^{\left(-\frac{t}{v}\right)}\left(\frac{t}{-}\right)^{p+b-1}\left(\frac{d t}{v}\right)
\end{aligned}
$$

Put $n=n-p$, we get

$$
\begin{aligned}
\mathrm{I} & =\frac{1}{\Gamma(a) \Gamma(b)} \sum_{m, n=0}^{\infty} \sum_{p=0}^{n} \frac{x^{m} y^{n-p} z^{p}}{(c)_{m}(d)_{n-p+p} m !(n-p) ! p !} \int_{0}^{\infty} \int_{0}^{\infty} e^{\left(-\frac{s-t}{u}-\frac{v}{v}\right)}\left(\frac{s}{u}\right)^{2 m+2 n-2 p+p+a-1}\left(\frac{t}{v}\right)^{b+p-1} \frac{d s}{u} \frac{d t}{v} \\
& =\frac{1}{\Gamma(a) \Gamma(b)} \sum_{m, n=0}^{\infty} \sum_{p=0}^{n} \frac{x^{m} y^{n-p} z^{p}}{(c)_{m}(d)_{n} m !(n-p) ! p !} \int_{0}^{\infty} \int_{0}^{\infty} e^{\left(-\frac{s-t}{u}-\frac{t}{v}\right)}\left(\frac{s}{u}\right)^{2 m+2 n-p+a-1}\left(\frac{t}{v}\right)^{b+p-1} \frac{d s}{u} \frac{d t}{v}
\end{aligned}
$$


Using the formula $\frac{1}{(n-p) !}=\frac{(-n)_{p}(-1)^{p}}{n !}$ we have

$\mathrm{I}=\frac{1}{\Gamma(a) \Gamma(b)} \sum_{p=0}^{n} \sum_{m, n=0}^{\infty} \frac{x^{m} y^{n-p} z^{p}(-n)_{p}(-1)^{p}}{(c)_{m}(d)_{n} m ! n ! p !} \int_{0}^{\infty} \int_{0}^{\infty} e^{\left(-\frac{s}{u}-\frac{t}{v}\right)}\left(\frac{s}{u}\right)^{2 m+2 n+a-1}\left(\frac{t}{v}\right)^{b-1}\left(\frac{t u}{s v}\right)^{p}\left(\frac{d s}{u}\right)\left(\frac{d t}{v}\right)$

$=\frac{1}{\Gamma(a) \Gamma(b)} \sum_{p=0}^{n} \sum_{m, n=0}^{\infty} \frac{x^{m} y^{n}(-n)_{p}(-1)^{p}}{(c)_{m}(d)_{n} m ! n ! p !} \frac{1}{u v} \int_{0}^{\infty} \int_{0}^{\infty} e^{\left(-\frac{s}{u}-\frac{t}{v}\right)}\left(\frac{s}{u}\right)^{a-1+2 m+2 n}\left(\begin{array}{c}t \\ \frac{v}{v}\end{array}\right)^{b-1}\left(\frac{z t u}{y s v}\right)^{p} d s d t$

$$
\sum_{p=0}^{n} \frac{(-n)_{p}(-1)^{p}}{p !}\left(\frac{z t u}{y s v}\right)^{p}=\left(1+\frac{z t u}{y s v}\right)^{n}=\left(\frac{y s v+z t u}{y s v}\right)^{n}
$$

Changing the order of series and integration, we get

$$
\begin{aligned}
\mathrm{I} & =\frac{1}{\Gamma(a) \Gamma(b)} \frac{1}{u v} \int_{0}^{\infty} \int_{0}^{\infty} e^{\left(-\frac{s}{u}-\frac{t}{v}\right)}\left(\frac{s}{u}\right)^{a-1}\left(\frac{t}{\frac{v}{v}}\right)^{b-1} \sum_{m, n=0}^{\infty} \frac{x^{m} y^{n}}{(c)_{m}(d)_{n} m ! n !}\left(\frac{s}{u}\right)^{2 m}\left(\frac{y s v+z t u}{y s v}\right)^{n}\left(\frac{s}{u}\right)^{2 n} d s d t \\
& =\frac{1}{\Gamma(a) \Gamma(b)} \frac{1}{u v} \int_{0}^{\infty} \int_{0}^{\infty} e^{\left(-\frac{s-t}{u}\right)}\left(\frac{s}{u}\right)^{a-1}\left(\frac{t}{u}\right)^{b-1} \sum_{m, n=0}^{\infty} \frac{x^{m}}{(c)_{m}(d)_{n} m ! n !}\left(\frac{s^{2}}{u^{2}}\right)^{m}\left(\frac{y s v+z t u}{s v}\right)^{n}\left(\frac{s^{2}}{u^{2}}\right)^{n} d s d t \\
& =\frac{1}{\Gamma(a) \Gamma(b)} \frac{1}{u v} \int_{0}^{\infty} \int_{0}^{\infty} e^{\left(-\frac{s}{u}-\frac{t}{v}\right)}\left(\frac{s}{-}\right)^{a-1}\left(\frac{t}{-}\right)^{b-1} \sum_{m=0}^{\infty} \frac{1}{(c)_{m} m !}\left(\frac{x s^{2}}{u^{2}}\right)^{m} \sum_{n=0}^{\infty} \frac{1}{(d)_{n} n !}\left(\frac{y s^{2} v+z s t u}{u^{2} v}\right)^{n} d s d t \\
& =\frac{1}{\Gamma(a) \Gamma(b)} \frac{1}{u v} \int_{0}^{\infty} \int_{0}^{\infty} e^{\left(-\frac{s}{u}-\frac{t}{v}\right)}\left(\frac{s}{u}\right)^{a-1}\left(\frac{t}{u}\right)^{b-1}{ }_{0} F_{1}\left(-; c ; \frac{x s^{2}}{u^{2}}\right)_{0} F_{1}\left(-; d ; \frac{y s^{2} v+z s t u}{u^{2} v}\right) d s d t \\
& =\text { R.H.S }
\end{aligned}
$$




\section{Special Case:}

If we put $u=1$ and $v=1$ in (4.1) then we get

$$
\begin{aligned}
& X_{1}(a, b ; c, d ; x, y, z) \\
& =\frac{1}{\Gamma(a) \Gamma(b)} \int_{0}^{\infty} \int_{0}^{\infty} e^{-s-t} s^{a-1} t^{b-1} F_{1}\left(-; c ; x s^{2}\right){ }_{0} F_{1}\left(-; d ; y s^{2}+z s t\right) d s d t
\end{aligned}
$$

This verifies the Exton result. Rest could be verified in the same manner.

\section{Acknowledgements}

Authors are thankful to the three independent reviewers for the fruitful comments and suggestions which improves the quality of the work.

\section{References}

[1] Agarwal R.P., K1licman A., Parmar R.K. and Rathie Arjun K.(2019). Certain generalized fractional calculus formulas and integral transforms involving $(p, q)$-Mathieu-type series , Advances in Difference Equations , 221 (2019). https://doi.org/10.1186/s13662-019-2142-0, Springer.

[2] Exton H.(1976). Hypergeometric function of three variables , Jour. Indian Acad. Maths ,4(2)

[3] Exton H. (1976). Handbook of Hypergeometric Integrals, Chichester: Ellis Horwood Ltd.

[4] Exton H. (1976). Multiple Hypergeometric Function \& Applications, Chichester: Ellis Horwood Ltd.

[5] Exton H. (1983). q-Hypergeometric Function \& Applications, Chichester: Ellis Horwood Ltd.

[6] Goswami P. and Belgacem F.B.M. (2012). Fractional differential equation solutions through a Sumudu rational, Nonlinear Studies, Academia.Ed, 19(4): 591-598

[7] Goyal R. and Purohit M. (2020). The Double Integral Transforms of the incomplete generalized SFunction, International Journal of Advanced Science and Technology, 29(11):3061-3067

[8] Lauricella G. (1893). Sulle funzioni ipergeometriche a più variabili, Rendiconti del Circolo Matematico di Palermo (in Italian), $7: 111-158$.

[9] Saran S. (1954). Hypergeometric functions of three variables, Ganita, 5 : 71-91.

[10] Srivastava H.M. and Karlsson P. W. (1985). Multiple Gaussian hypergeometric series. Mathematics and its applications. Chichester, UK: Halsted Press, Ellis Horwood Ltd.

[11] Srivastava H.M. and Kashyap B.R.K. (1982). Special Functions in Queuing Theory and Related Stochastic Processes, Academic Press, New York. 